\title{
Manual of Digital Earth
}

Guo, H., Goodchild, M.F. \& Annoni, A. (eds.), Singapore, Springer, 2020, 852 pp. ISBN: 978-981-32-9914-6. DOI https://doi.org/10.1007/978-981-32-9915-3

\section{Luis M. Vilches-Blázquez Instituto Politécnico Nacional, México}

El concepto de la Tierra Digital (Digital Earth) fue acuñado por primera vez en el libro de Al Gore titulado "Earth in the Balance" (Gore, 1992). En 1999, la Tierra Digital fue concebida como una representación tridimensional del planeta con múltiples resoluciones que permitirían buscar, visualizar y poner en valor grandes cantidades de información georreferenciada en entornos físicos y sociales. Dicho sistema permitiría a los usuarios navegar a través del espacio y tiempo, acceder a datos históricos, así como a predicciones futuras, y su uso sería promovido, de igual forma, por científicos, responsables políticos y niños (Gore, 1999).

Este concepto fue motivado por la idea de que las preguntas complejas no se pueden responder desde un solo dominio, sino que se necesitan múltiples disciplinas (Janowicz \& Hitzler, 2012). Así, la Tierra Digital trajo consigo la visión de una plataforma común que apoyaría la cooperación nacional e internacional para el desarrollo sostenible global y que conllevaría un nuevo punto de crecimiento económico y bienestar social (International Society for Digital Earth, 2012).

Tras cumplirse dos décadas de este concepto (Digital Earth), se han logrado muchos avances, definiendo estándares, implementando prototipos, popularizando productos de la industria y creando aplicaciones a través de iniciativas en todo el mundo (Yang et al., 2010). Muchos de los elementos no solo están disponibles y son utilizados diariamente por cientos de millones de personas en todo el mundo (Craglia et al., 2008), sino que se están convirtiendo en un elemento clave para promover una comprensión y análisis global (Guo, 2016). De esta manera, la consolidación y avance de los elementos de la Tierra Digital están contribuyendo a la implementación exitosa de la Tierra del Futuro (Future Earth) (Songnian \& Jun, 2017).

Tomando en cuenta este contexto, este manual, elaborado con la participación de más de 100 colaboradores y editores de 18 países, aborda diversas preguntas con respecto a la relevancia de la Tierra Digital, su futuro y su potencial para apoyar el desarrollo científico y el progreso social. Así, el manual presenta la siguiente estructura: 
- El capítulo de introducción proporciona una visión general de la Tierra Digital y su marco de referencia, su respuesta global, evolución y relación con iniciativas científicas globales, así como la respuesta a desafíos globales que son detallados en capítulos posteriores.

- La Parte I, presenta uno de los puntos claves en este libro, asociado a una visión general sobre conceptos, teorías y métodos fundamentales relacionados con la Tierra Digital, como son: los Sistema de Mallas Globales y Discretas (DGGS), sensores remotos, sistema global de navegación por satélite (GNSS), infraestructuras de información geoespacial, zoom continuo y transformación multi-escala, Big Data y computación en la nube, inteligencia artificial y técnicas de aprendizaje automático, Internet de las cosas (IoT) y redes sociales. Además, esta parte describe algunas tecnologías para el procesamiento de datos geoespaciales (computación de alto rendimiento y geoprocesamiento en línea y distribuido), y proporciona detalles sobre geovisualización y realidad extendida (realidad virtual, aumentada y mixta) con ejemplos realmente interesantes. Cada uno de estos temas está organizado como un capítulo independiente.

- La Parte II describe diversas aplicaciones de la nueva generación de sistemas de la Tierra Digital que tienen como objetivo satisfacer la demanda de intercambio de información para su contribución en diferentes escenarios. Por lo tanto, un gran número de estos capítulos ofrecen evidencia del potencial, limitaciones y desafíos de la Tierra Digital y, más específicamente, sobre su nueva visión denominada Big Earth Data (Guo, 2016). Así, se describe el nuevo entorno para generar, compartir e integrar datos, información y conocimientos multidimensionales, multiescalares y multitemporales en apoyo de los Objetivos de Desarrollo Sostenible $\mathrm{y}$, más concretamente, en aquellos escenarios relacionados con el cambio climático, mitigación de desastres y ciudades y comunidades sostenibles. Además, esta parte reúne tres capítulos de especial interés que discuten el patrimonio digital, ciencia ciudadana y aspectos económicos. El primero muestra cómo se utilizan los medios digitales para preservar, proteger, estudiar y presentar el patrimonio natural y cultural. En el segundo, se aborda una visión general del concepto de ciencia ciudadana, sus prácticas tecnológicas e impactos sociales, así como sus desafíos, mientras el último se centra en el valor económico de la Tierra Digital que, según los autores, depende de perspectivas asociadas con para quién, con qué propósito y con cuándo.

- La Parte III, aglutina las contribuciones de una serie de regiones a los objetivos de la Tierra Digital. De este modo, el manual destaca el establecimiento de la Infraestructura Europea de Datos Espaciales, los programas Galileo y Copernicus, las plataformas basadas en la nube y el panorama de la ciencia ciudadana en el caso europeo. Con respecto a las propuestas australianas, se discuten diversas iniciativas nacionales (por ejemplo, Geoscape, Australian Geoscience Data Cube 
o Digital Earth Australia) y se proporcionan casos de uso en varios dominios, lecciones aprendidas y oportunidades para este país. En China, el desarrollo de las tecnologías y aplicaciones de la Tierra Digital se ha basado en estrategias y políticas, respaldadas por tecnologías de red e información (por ejemplo, 5G, IPV6, infraestructura de computación en la nube o plataformas de Big Data), que se han utilizado en algunas ciudades y aplicaciones. Finalmente, una serie de datos e iniciativas muestran una consolidada comunidad en Rusia que presenta incluso antecedentes históricos.

- La Parte IV de este manual proporciona una visión general de las estructuras actuales de enseñanza y aprendizaje donde la Tierra Digital está presente y destaca la importancia de un marco de aprendizaje integrado para una mejor preparación de (futuros) estudiantes en el ámbito geoespacial. Además, se tratan los códigos éticos asociados con la (geo) privacidad y la dignidad humana, basándose en los escenarios de la legislación de Europa y Estados Unidos, como referencia, aunque se realizan menciones a la legislación y directrices del continente africano y China.

- Finalmente, el libro concluye con la revisión del pasado y presente de la Tierra Digital, seguido de la presentación de un conjunto de desafíos y tendencias futuras asociadas con la nueva era del Big Earth Data. Adicionalmente, los autores especulan sobre cómo la Tierra Digital puede evolucionar en los próximos años en función de los impulsores científicos, avances tecnológicos, adopción de aplicaciones y la construcción de una comunidad (virtual y física) relevante.

En conclusión, recomiendo este libro a todos los lectores de la Revista Cartográfica y a aquellos investigadores, estudiantes y tomadores de decisiones relacionados o interesados en la Tierra Digital, así como al conjunto de la comunidad geoespacial. Las más de 800 páginas que componen esta obra recogen el contenido más actualizado, a la fecha, y uno de los compendios más detallados sobre esta disciplina promovido por la Sociedad Internacional para la Tierra Digital (International Society for Digital Earth - ISDE). Por todo lo anterior, este libro se convierte en una obra de referencia y, en consecuencia, merece un lugar en cada biblioteca universitaria y en las estanterías de aquellos profesionales que trabajan en el ámbito geoespacial.

\section{Bibliografía}

Craglia, M., Goodchild, M.F., Annoni, A., Camara, G., Gould, M., Kuhn, W. \& Parsons, E. (2008). "Next-generation Digital Earth. A position paper from the Vespucci Initiative for the Advancement of Geographic Information Science", International Journal of Spatial Data Infrastructure Research, 3(3): 146-167.

Gore, A. (1992). Earth in the balance: ecology and the human spirit, Houghton Mifflin, Boston. 
(1999). "The Digital Earth: Understanding Our Planet in the 21st Century" Photogrammetric Engineering and Remote Sensing, 65(5): 528.

Guo, H. (2016). "Digital Earth and Future Earth", International Journal of Digital Earth, 9(1): 1-2. https://doi.org/10.1080/17538947.2015.1135667

International Society for Digital Earth (2012). Statutes of the International Society for Digital Earth. http://www.digitalearth-isde.org/statues

Janowicz, K. \& Hitzler, P. (2012). The Digital Earth as Knowledge Engine (editorial). Semantic Web Journal, 3(3): 213-221. https://doi.org/10.3233/SW-2012$\underline{0070}$

Songnian, L. \& Jun, C. (2017). Supporting future earth with global geospatial information, International Journal of Digital Earth, 10(4): 325-327. https://doi.org/10.1080/17538947.2016.1275832

Yang, C., Raskin, R., Goodchild, M. \& Gahegan, M. (2010). "Geospatial Cyberinfrastructure: Past, Present, and Future". Computers, Environment and Urban Systems, 34 (4): 264-277. https://doi.org/doi:10.1016/j.compenvurbsys.2010.04.001 